\title{
A New Two - Parameter Lindley Distribution
}

\author{
Rama Shanker \& Umme Habibah Rahman \\ Department of Statistics, Assam University, Silchar, India \\ Email: shankerrama2009@gmail.com; umme.habibah.rahman17@gmail.com
}

Corresponding Author: Rama Shanker

\begin{abstract}
In this paper, a new two - parameter Lindley distribution has been proposed. Descriptive statistical properties along with order statistics, Fisher information matrix and confidence interval of the proposed distribution have been discussed. Parameters are estimated by the method of Maximum Likelihood estimation. A real lifetime data has been presented to test the goodness of fit of the proposed distribution over other one parameter and two -parameter Lindley family of distributions.
\end{abstract}

Keywords: Lindley distribution, Two-parameter Lindley distributions, Statistical properties, Maximum likelihood estimation, Fisher information matrix.

\section{Introduction}

The exponential distribution and the Lindley distribution were the two classical lifetime distributions for modeling lifetime data. Lindley [5] introduced a lifetime distribution known as Lindley distribution defined by the probability density function (pdf) and cumulative distribution function (cdf)

$$
\begin{gathered}
f(x ; \theta)=\frac{\theta^{2}}{\theta+1}(1+x) e^{-\theta x} ; x>0 \text { and } \theta>0 \\
F(x ; \theta)=1-\left[1+\frac{\theta x}{\theta+1}\right] e^{-\theta x} ; x>0 \text { and } \theta>0
\end{gathered}
$$

Lindley distribution, being a convex combination of exponential and gamma distribution, gives better fit than exponential distribution and it is more flexible than the exponential distribution. Ghitany et al [3] have studied many interesting properties, estimation of parameter using both the method of moments and the method of maximum likelihood, and application of Lindley distribution. Recently several twoparameter Lindley distributions have been introduced by different researchers. Some important twoparameter lifetime distribution proposed by different researchers are presented in the following Table 1.

Table 1: Two-parameter Lindley distributions with their pdf and introducer (year).

\begin{tabular}{|c|c|c|}
\hline $\begin{array}{c}\text { Name of the } \\
\text { distributions }\end{array}$ & probability density function (pdf) & Introducers \\
\hline $\begin{array}{c}\text { Two-parameter } \\
\text { Lindley distribution- } \\
1 \text { (TPLD-1) }\end{array}$ & $f(x ; \theta, \alpha)=\frac{\theta^{2}}{\theta \alpha+1}(\alpha+x) e^{-\theta x} ; x>0, \theta>0, \theta \alpha>-1$ & $\begin{array}{c}\text { Shanker and } \\
\text { Mishra } \\
\text { (2013)[8] }\end{array}$ \\
\hline
\end{tabular}




\begin{tabular}{|c|c|c|}
\hline $\begin{array}{c}\text { Two-parameter } \\
\text { Lindley distribution- } \\
\text { 2 (TPLD-II) }\end{array}$ & $f(x ; \theta, \alpha)=\frac{\theta^{2}}{\theta+\alpha}(1+\alpha x) e^{-\theta x} ; x>0, \theta>0, \alpha>0$ & $\begin{array}{c}\text { Shanker } \text { et al } \\
\text { (2013)[7] }\end{array}$ \\
\hline $\begin{array}{c}\text { Quasi Lindley } \\
\text { distribution (QLD) }\end{array}$ & $f(x ; \theta, \alpha)=\frac{\theta}{\alpha+1}(\alpha+\theta x) e^{-\theta x} ; x>0, \theta>0, \alpha>-1$ & $\begin{array}{c}\text { Shanker and } \\
\text { Mishra } \\
\text { (2013)[9] }\end{array}$ \\
\hline $\begin{array}{c}\text { New Quasi Lindley } \\
\text { distribution (NQLD) }\end{array}$ & $f(x ; \theta, \alpha)=\frac{\theta^{2}}{\theta^{2}+\alpha}(\theta+\alpha x) e^{-\theta x} ; x>0, \theta>0, \alpha>0$ & $\begin{array}{c}\text { Shanker and } \\
\text { Amanuel } \\
\text { (2013)[10] }\end{array}$ \\
\hline
\end{tabular}

The statistical properties, estimation of parameters using both the method of moments and maximum likelihood estimation and applications of these two-parameter Lindley distributions are available in the respective papers. Some of the important generalizations and extensions of Lindley distribution have been done by researchers including Bakouch et al [2] and Ghitany et al [4], Nadarajah et al [6], Zakerzadeh and Dolati [11], some among others.

The main purpose of this paper is to introduce a new two-parameter Lindley distribution which gives much better fit than the existing two-parameter Lindley distributions in statistics literature. Several properties like reliability analysis, mean residual life function and moments have been discussed. Estimation of parameters is estimated by the method of maximum likelihood and Fisher information matrix with confidence interval is also given. At last, goodness of fit of the proposed distribution and its comparative fit with other one and two-parameter Lindley family of distributions are discussed.

\section{A New Two-Parameter Lindley Distribution}

The pdf and the cdf of the new two-parameter Lindley distribution (NTPLD) can be expressed as

$$
\begin{gathered}
f(x ; \alpha, \theta)=\frac{\theta^{\alpha+1}\left(\alpha+x^{\alpha}\right) e^{-\theta x}}{\alpha \theta^{\alpha}+\Gamma(\alpha)} ; x>0 ; \alpha>0 \text { and } \theta>0, \\
F(x ; \alpha, \theta)=\frac{\alpha \theta^{\alpha}\left(1-e^{-\theta x}\right)+\gamma(\alpha+1, \theta x)}{\alpha \theta^{\alpha}+\Gamma(\alpha+1)} ; x>0 ; \alpha>0 \text { and } \theta>0 .
\end{gathered}
$$

where $\alpha$ is the shape parameter and $\theta$ is the scale parameter and $\gamma(\alpha, z)=\int_{0}^{z} e^{-t} t^{\alpha-1} d t$ is the lower incomplete gamma function. The NTPLD has a proper density function since

$$
\lim _{x \rightarrow \infty} F(x ; \alpha, \theta)=1 \quad \text { and } \quad \lim _{x \rightarrow-\infty} F(x ; \alpha, \theta)=0
$$

Graphs of the pdf and cdf of NTPLD has shown in figures 1 and 2 respectively for varying values of the parameters $\alpha$ and $\theta$. 

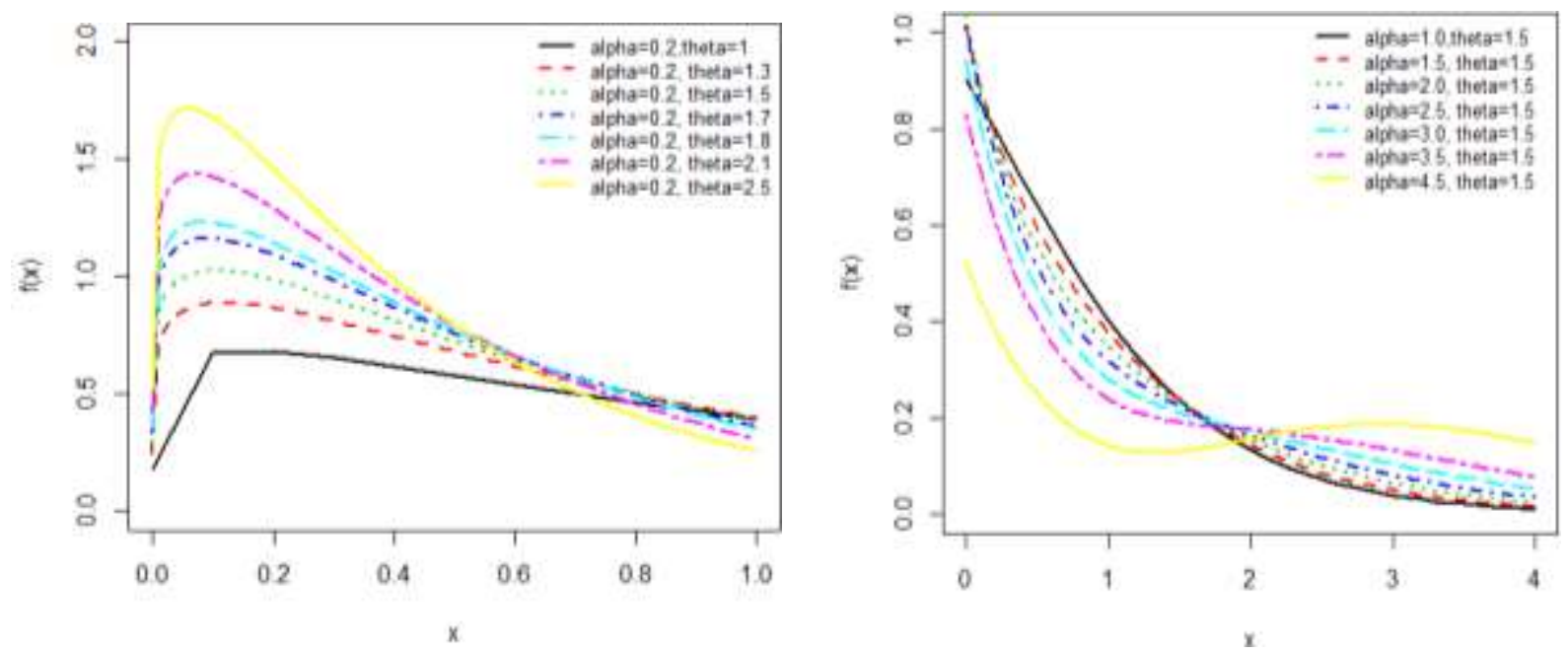

Fig.1: pdf plot of NTPLD for varying values of parameters
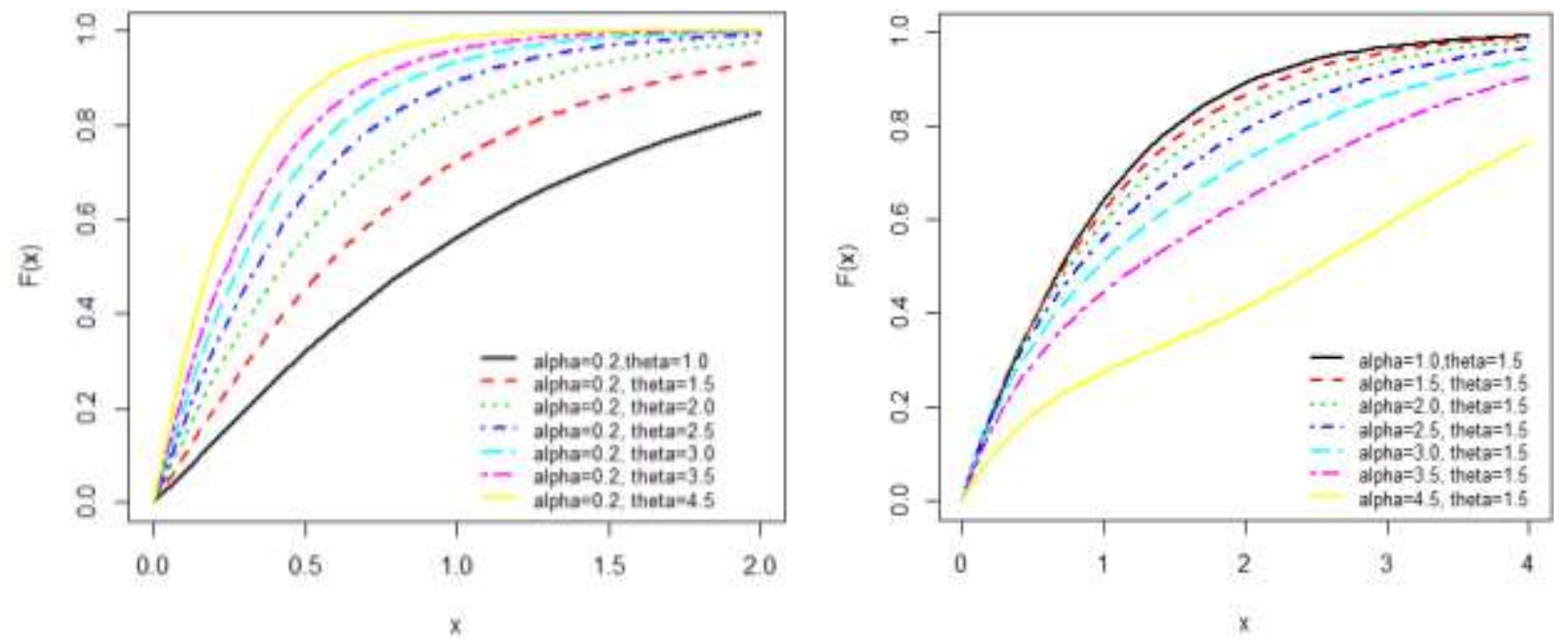

Fig.2: cdf plot of NTPLD for varying values of parameters

\section{Statistical Properties}

In this section, statistical properties including asymptotic behavior, mean residual life function, reliability analysis of NTPLD have been studied.

\subsection{Asymptotic Behavior}

The asymptotic behavior of NTPLD for $x \rightarrow 0$ and $x \rightarrow \infty$ are

$$
\lim _{x \rightarrow 0} f(x ; \alpha, \theta)=\lim _{x \rightarrow 0}\left[\frac{\theta^{\alpha+1}\left(\alpha+x^{\alpha}\right) \exp (-\theta x)}{\alpha \theta^{\alpha}+\Gamma(\alpha)}\right]=0
$$




$$
\lim _{x \rightarrow \infty} f(x ; \alpha, \theta)=\lim _{x \rightarrow \infty}\left[\frac{\theta^{\alpha+1}\left(\alpha+x^{\alpha}\right) \exp (-\theta x)}{\alpha \theta^{\alpha}+\Gamma(\alpha)}\right]=0 .
$$

These results confirm that the proposed distribution has a mode.

\subsection{Reliability Analysis}

The survival function (or the reliability function) is the probability that a subject survives longer than the expected time. The survival function of NTPLD is given by

$$
S(x ; \alpha, \theta)=1-F(x ; \alpha, \theta)=\frac{\alpha \theta^{\alpha} e^{-\theta x}-\gamma(\alpha+1, \theta x)+\Gamma(\alpha+1)}{\alpha \theta^{\alpha}+\Gamma(\alpha+1)}
$$

The hazard function (also known as the hazard rate, instantaneous failure rate or force of mortality) is the probability to measure the instant death rate of a subject. Suppose $X$ be a continuous random variable with pdf $f(x)$ and cdf $F(x)$. The hazard rate function of $X$ is defined as

$$
h(x)=\lim _{\Delta x \rightarrow 0} \frac{P(X<x+\Delta x / X>x)}{\Delta x}=\frac{f(x)}{1-F(x)}
$$

The corresponding $h(x)$ of NTPLD can be obtained as

$$
h(x ; \alpha, \theta)=\frac{\theta^{\alpha+1}\left(\alpha+x^{\alpha}\right) e^{-\theta x}}{\alpha \theta^{\alpha} e^{-\theta x}-\gamma(\alpha+1, \theta x)+\Gamma(\alpha+1)}
$$

The natures of survival function and the hazard function of NTPLD for varying values of parameters are shown graphically in figures 3 and 4 , respectively.
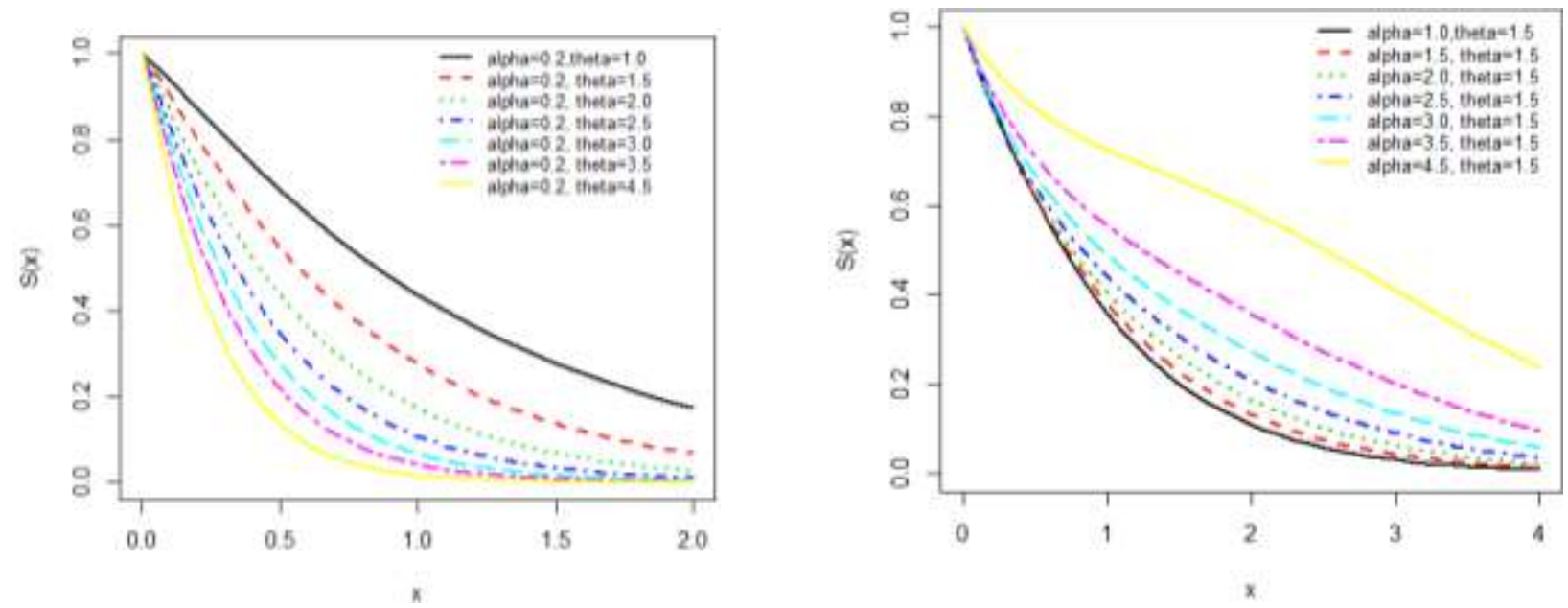

Fig.3: Survival function of NTPLD for varying values of parameters. 

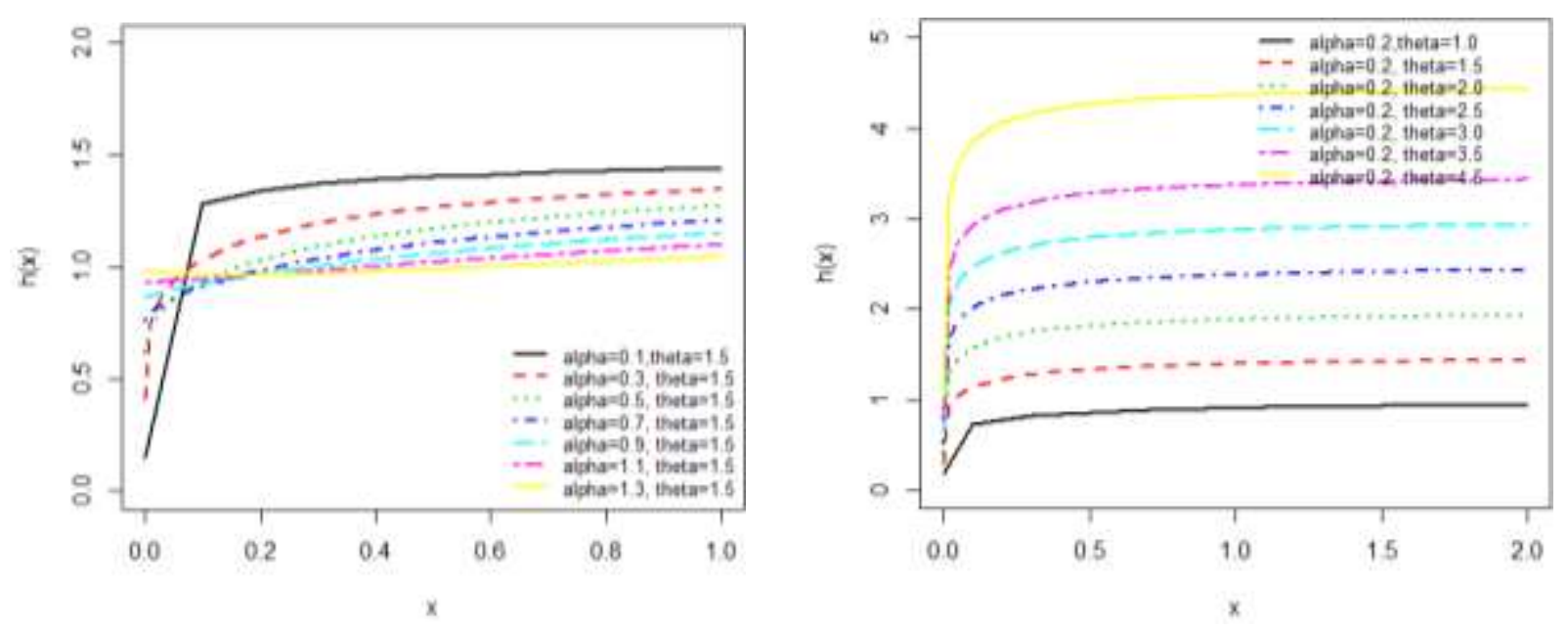

Fig.4: Hazard function of NTPLD for varying values of parameters.

\subsection{Mean Residual Life Function}

The mean residual life function of the NTPLD can be obtained as

$$
\begin{aligned}
m(x ; \alpha, \theta)=E[X-x \backslash X>x] & =\frac{1}{1-F(x ; \alpha, \theta)} \int_{x}^{\infty}[1-F(t ; \alpha, \theta)] d t \\
& =\frac{\alpha \theta^{\alpha-1} \gamma(\alpha, \theta x)+\gamma(\alpha+1, \theta x)}{\alpha \theta^{\alpha} e^{-\theta x}-\gamma(\alpha+1, \theta x)+\Gamma(\alpha+1)}-x
\end{aligned}
$$

\subsection{Moments and Related Measures}

The $r^{\text {th }}$ moment about origin of NTPLD has been obtained as

$$
\mu_{r}^{\prime}=\frac{\alpha \theta^{\alpha} \Gamma(\alpha+1)+\Gamma(r+\alpha+1)}{\theta^{r}\left[\alpha \theta^{\alpha}+\Gamma(\alpha+1)\right]} ; r=1,2, \ldots
$$

Taking $r=1,2,3$ and 4 in (3.4.1), the first four moments about origin are obtained as

$$
\begin{array}{ll}
\mu_{1}^{\prime}=\frac{\alpha \theta^{\alpha} \Gamma(\alpha+1)+\Gamma(\alpha+2)}{\theta\left[\alpha \theta^{\alpha}+\Gamma(\alpha+1)\right]} \quad \mu_{2}^{\prime}=\frac{\alpha \theta^{\alpha} \Gamma(\alpha+1)+\Gamma(\alpha+3)}{\theta^{r}\left[\alpha \theta^{\alpha}+\Gamma(\alpha+1)\right]} \\
\mu_{3}^{\prime}=\frac{\alpha \theta^{\alpha} \Gamma(\alpha+1)+\Gamma(\alpha+4)}{\theta^{3}\left[\alpha \theta^{\alpha}+\Gamma(\alpha+1)\right]} \quad \mu_{4}^{\prime}=\frac{\alpha \theta^{\alpha} \Gamma(\alpha+1)+\Gamma(\alpha+5)}{\theta^{4}\left[\alpha \theta^{\alpha}+\Gamma(\alpha+1)\right]}
\end{array}
$$

The first two central moments of NTPLD have been obtained as

$$
\begin{gathered}
\mu_{1}=0 \\
\mu_{2}=\frac{\alpha^{2} \theta^{2 \alpha}+\alpha \theta^{\alpha} \Gamma(\alpha+1)\left(\alpha^{2}+\alpha+2\right)+(\alpha+1) \Gamma^{2}(\alpha+1)}{\theta^{2}\left[\alpha \theta^{\alpha}+\Gamma(\alpha+1)\right]^{2}}
\end{gathered}
$$


The standard deviation and the coefficient of variation and coefficient of dispersion of NTPLD are obtained as

$$
\begin{gathered}
\text { Standard deviation }(\sigma)=\frac{\sqrt{\alpha^{2} \theta^{2 \alpha}+\alpha \theta^{\alpha} \Gamma(\alpha+1)\left(\alpha^{2}+\alpha+2\right)+(\alpha+1) \Gamma^{2}(\alpha+1)}}{\theta\left[\alpha \theta^{\alpha}+\Gamma(\alpha+1)\right]} \\
\text { Co-efficient of variation }\left(\frac{\sigma}{\mu_{1}^{\prime}}\right)=\frac{\sqrt{\alpha^{2} \theta^{2 \alpha}+\alpha \theta^{\alpha} \Gamma(\alpha+1)\left(\alpha^{2}+\alpha+2\right)+(\alpha+1) \Gamma^{2}(\alpha+1)}}{\alpha \theta^{\alpha} \Gamma(\alpha+1)+\Gamma(\alpha+2)} \\
\text { Co-efficient of dispersion }\left(\frac{\sigma^{2}}{\mu_{1}^{\prime}}\right)=\frac{\alpha^{2} \theta^{2 \alpha}+\alpha \theta^{\alpha} \Gamma(\alpha+1)\left(\alpha^{2}+\alpha+2\right)+(\alpha+1) \Gamma^{2}(\alpha+1)}{\theta\left[\alpha \theta^{\alpha}+\Gamma(\alpha+1)\right]\left[\alpha \theta^{\alpha} \Gamma(\alpha+1)+\Gamma(\alpha+2)\right]}
\end{gathered}
$$

The forms of the rest central moments and related measure like kurtosis and skewness are very big and complicated and thus are not given here. However, if required, can be obtained in the same way.

\section{Generating Functions}

Let $X$ have a NTPLD, then the MGF of $X$ is obtained as

$$
M_{X}(t)=E\left(e^{t X}\right)=\int_{0}^{\infty} e^{t x} f(x) d x
$$

Using Taylor's series, we have

$$
\begin{aligned}
M_{X}(t) & =E\left(e^{t x}\right)=\int_{0}^{\infty}\left(1+t x+\frac{(t x)^{2}}{2 !}+\cdots\right) f(x) d x \\
& =\int_{0}^{\infty} \sum_{j=0}^{\infty} \frac{t^{j}}{j !} x^{j} g(x) d x=\sum_{j=0}^{\infty} \frac{t^{j}}{j !} \mu_{j}{ }^{\prime}
\end{aligned}
$$

The moment generating function of NTPLD is given by

$$
M_{X}(t)=\sum_{j=0}^{\infty} \frac{t^{j}}{j !} \frac{\alpha \theta^{\alpha} \Gamma(\alpha+1)+\Gamma(j+\alpha+1)}{\theta^{j}\left[\alpha \theta^{\alpha}+\Gamma(\alpha+1)\right]}
$$

Similarly, the characteristic function of NTPLD can be obtained as

$$
\Phi_{X}(t)=M_{X}(i t)=\sum_{j=0}^{\infty} \frac{(i t)^{j}}{j !} \frac{\alpha \theta^{\alpha} \Gamma(\alpha+1)+\Gamma(j+\alpha+1)}{\theta^{j}\left[\alpha \theta^{\alpha}+\Gamma(\alpha+1)\right]}
$$

The cumulant generating function is given by

$$
K_{X}(t)=\ln \left[M_{X}(i t)\right]=\ln \left[\sum_{j=0}^{\infty} \frac{(i t)^{j}}{j !} \frac{\alpha \theta^{\alpha} \Gamma(\alpha+1)+\Gamma(j+\alpha+1)}{\theta^{j}\left[\alpha \theta^{\alpha}+\Gamma(\alpha+1)\right]}\right]
$$




\section{Distribution of Order Statistics}

Let $x_{1}, x_{2}, \ldots, x_{n}$ be the random samples from $\operatorname{NTPLD}(\alpha, \theta)$. The pdf of $i^{\text {th }}$ order statistics is given by

$$
f_{i: n}(x)=\frac{n !}{(i-1) !(n-i) !} f_{X}(x)\left[F_{X}(x)\right]^{i-1}\left[1-F_{X}(x)\right]^{n-i}
$$

The pdf of $i^{\text {th }}$ order statistics $X_{(i)}$ of NTPLD is given by

$$
\begin{aligned}
f_{i: n}(x)=\frac{n !}{(i-1) !(n-i) !} & \frac{\theta^{\alpha+1}\left(\alpha+x^{\alpha}\right) \exp (-\theta x)}{\alpha \theta^{\alpha}+\Gamma(\alpha)}\left[\frac{\alpha \theta^{\alpha}\left(1-e^{-\theta x}\right)+\gamma(\alpha+1, \theta x)}{\alpha \theta^{\alpha}+\Gamma(\alpha+1)}\right]^{i-1} \\
\times & {\left[1-\frac{\alpha \theta^{\alpha}\left(1-e^{-\theta x}\right)+\gamma(\alpha+1, \theta x)}{\alpha \theta^{\alpha}+\Gamma(\alpha+1)}\right]^{n-i} }
\end{aligned}
$$

The pdf of the first order statistic $X_{(1)}$ can be expressed as

$$
f_{1: n}(x)=n \frac{\theta^{\alpha+1}\left(\alpha+x^{\alpha}\right) \exp (-\theta x)}{\alpha \theta^{\alpha}+\Gamma(\alpha)}\left[1-\frac{\alpha \theta^{\alpha}\left(1-e^{-\theta x}\right)+\gamma(\alpha+1, \theta x)}{\alpha \theta^{\alpha}+\Gamma(\alpha+1)}\right]^{n-1}
$$

The pdf of the largest order statistic $X_{(n)}$ can be expressed as

$$
f_{n: n}(x)=n \frac{\theta^{\alpha+1}\left(\alpha+x^{\alpha}\right) \exp (-\theta x)}{\alpha \theta^{\alpha}+\Gamma(\alpha)}\left[\frac{\alpha \theta^{\alpha}\left(1-e^{-\theta x}\right)+\gamma(\alpha+1, \theta x)}{\alpha \theta^{\alpha}+\Gamma(\alpha+1)}\right]^{n-1}
$$

\section{Maximum Likelihood Estimation}

Let $x_{1}, x_{2}, \ldots, x_{n}$ be a random sample of size $n$ from a $\operatorname{NTPLD}(\alpha, \theta)$. The log-likelihood function can be expressed as

$$
\log L=n\left[(\alpha+1) \ln \theta-\ln \left\{\alpha \theta^{\alpha}+\Gamma(\alpha+1)\right\}\right]+\sum_{i=1}^{n} \ln \left(\alpha+x_{i}^{\alpha}\right)-n \theta \bar{x}
$$

The maximum likelihood estimates (MLE) $(\hat{\alpha}, \hat{\theta})$ of parameters $(\alpha, \theta)$ of NTPLD are the solutions of the following log- likelihood equations

$$
\begin{gathered}
\frac{\partial}{\partial \alpha} \log L=n\left[\ln \theta-\frac{1}{\alpha \theta^{\alpha}+\Gamma(\alpha+1)}\left\{\alpha^{2} \theta^{\alpha-1}+\theta^{\alpha}+\Psi(\alpha+1)\right\}\right]+\frac{n}{\alpha}+\frac{\alpha \sum_{i=1}^{n} x_{i}^{\alpha-1}}{\sum_{i=1}^{n} x_{i}^{\alpha}} \\
\frac{\partial}{\partial \theta} \log L=\frac{n(\alpha+1)}{\theta}-\frac{n \alpha^{2} \theta^{\alpha-1}}{\alpha \theta^{\alpha}+\Gamma(\alpha+1)}-n \bar{x}
\end{gathered}
$$




$$
\text { where } \Psi(\alpha+1)=\frac{\partial}{\partial \alpha} \Gamma(\alpha+1)
$$

These log-likelihood equation can not be solved analytically and required statistical software with iterative numerical techniques. These equations can be solved using R-software.

The $2 \times 2$ observed information matrix of NTPLD can be presented as,

$$
\left.\left(\begin{array}{l}
\hat{\alpha} \\
\hat{\theta}
\end{array}\right) \sim\left(\begin{array}{l}
\alpha \\
\theta
\end{array}\right),\left(\begin{array}{c}
\frac{\partial^{2} \log L}{\partial \alpha^{2}} \frac{\partial^{2} \log L}{\partial \alpha \partial \theta} \\
\frac{\partial^{2} \log L}{\partial \theta \partial \alpha} \frac{\partial^{2} \log L}{\partial \theta^{2}}
\end{array}\right)\right]
$$

The inverse of the information matrix results in the well-known variance-covariance matrix. The $2 \times 2$ approximate Fishar information matrix corresponding to the above observed information matrix is given by

$$
I^{-1}=-E\left[\begin{array}{l}
\frac{\partial^{2} \log L}{\partial \alpha^{2}} \frac{\partial^{2} \log L}{\partial \alpha \partial \theta} \\
\frac{\partial^{2} \log L}{\partial \theta \partial \alpha} \frac{\partial^{2} \log L}{\partial \theta^{2}}
\end{array}\right]
$$

The solution of the Fisher information matrix will yield asymptotic variance and covariance of the ML estimators for $(\hat{\alpha}, \hat{\theta})$. The approximate $100(1-\alpha) \%$ confidence intervals for $(\alpha, \theta)$ respectively are $\hat{\alpha} \pm \mathrm{Z}_{\frac{\alpha}{2}} \frac{\sigma_{\alpha \alpha}}{n}$ and $\hat{\theta} \pm \mathrm{Z}_{\frac{\alpha}{2}} \frac{\sigma_{\theta \theta}}{n}$, where $\mathrm{Z}_{\alpha}$ is the upper $100 \alpha^{\text {th }}$ percentile of the standard normal distribution.

\section{Applications}

The new two parameter lindley distribution(NTPLD) has been fitted to a lifetime data-set. In this section, we present the fit of NTPLD and compare its goodness of fit with Weibull distribution (WD), two parameter Lindley-1 (TPLD-1), two parameter Lindley-2 (TPLD-2), Quasi-Lindley distribution (QLD), new Quasi-Lindley distribution (NQLD), Lindley distribution (LD) and exponential distribution (ED). The following dataset has been considered.

The data set, strength data, which were originally reported by Badar and Priest [1] and it represents the strength measured in GPA for single carbon fibers and impregnated 1000-carbon fiber tows. Single fibers were tested under tension at gauge lengths of $10 \mathrm{~mm}$ with sample size $(n=63)$. This data set consists of observations:

$1.901,2.132,2.203,2.228,2.257,2.350,2.361,2.396,2.397,2.445,2.454,2.474,2.518,2.522,2.525$, $2.532,2.575,2.614,2.616,2.618,2.624,2.659,2.675,2.738,2.740,2.856,2.917,2.928,2.937,2.937$, 
2.977, 2.996, 3.030, 3.125, 3.139, 3.145, 3.220, 3.223, 3.235, 3.243, 3.264, 3.272, 3.294, 3.332, 3.346, $3.377,3.408,3.435,3.493,3.501,3.537,3.554,3.562,3.628,3.852,3.871,3.886,3.971,4.024,4.027$, $4.225,4.395,5.020$.

In order to compare NTLD with other distributions, we consider the criteria like Bayesian Information Criterion (BIC), Akaike Information Criterion (AIC), Akaike Information Criterion Corrected (AICC) and $-2 \log L$. The better distribution corresponds to lesser values of AIC, BIC, AICC and $-2 \log L$. The formulae for calculating AIC, BIC and AICC are as follows:

$$
A I C=2 k-2 \log L, B I C=k \log n-2 \log L, A I C C=A I C+\frac{2 k(k+1)}{(n-k-1)},
$$

where $k$ is the number of parameters, $n$ is the sample size and $-2 \log L$ is the maximized value of $\log$ likelihood function. The ML estimates of the parameters of the considered distributions along with values of $-2 \log L, A I C, A I C C$ and $B I C$ for the datasets are presented in Table 1.

Table 1: ML estimates of the parameters of the considered distributions along with values of $-2 \log L, A I C, A I C C, B I C$

\begin{tabular}{|c|c|c|c|c|c|c|}
\hline \multirow{2}{*}{ Distributions } & \multicolumn{2}{|c|}{ ML parameters } & $-2 \log L$ & \multirow{2}{*}{ AIC } & AICC & \multirow{2}{*}{ BIC } \\
\cline { 2 - 6 } & $\alpha$ & $\boldsymbol{\theta}$ & & & & \\
\hline NTPLD & 32.35558 & 10.50217 & 123.9867 & 127.9867 & 128.1867 & 132.2730 \\
\hline WD & 0.100000 & 2.244289 & 179.8587 & 183.8587 & 184.0587 & 188.1449 \\
\hline TPLD-1 & 2.295784 & 6.536456 & 220.7134 & 224.7134 & 224.9134 & 228.9997 \\
\hline TPLD-2 & 0.100000 & 0.634249 & 224.3504 & 228.3504 & 228.5504 & 232.6366 \\
\hline QLD & 0.100000 & 0.637224 & 226.2462 & 230.2462 & 230.4462 & 234.5325 \\
\hline NQLD & 2179.448 & 0.653649 & 220.7080 & 224.7080 & 224.9080 & 228.9943 \\
\hline LD & 0.539233 & - & 242.7152 & 244.7153 & 245.1220 & 246.8584 \\
\hline ED & 0.326873 & - & 266.8916 & 268.8915 & 269.2988 & 271.0347 \\
\hline
\end{tabular}

It is obvious from the goodness of fit of the two - parameter and one parameter distributions in table 1 that NTPLD gives much closer fit than other two parameter distributions.

\section{Conclusions}

A new two-parameter Lindley distribution (NTPLD) has been proposed. The nature of pdf, cdf, survival function, hazard rate function has been studied with varying values of parameters. Descriptive measures based on moments of NTPLD has been derived. The method of maximum likelihood has been discussed for estimating parameters. Fisher's information matrix and confidence intervals of the parameters of the proposed distribution have been presented. The goodness of fit of NTPLD has been discussed with a real 


\section{Rama Shanker and Umme Habibah Rahman/A New Two - Parameter Lindley Distribution}

lifetime data and compared with other two - parameter Lindley distribution and one parameter Lindley distribution and exponential distribution.

\section{Acknowledgements}

Authors are grateful for the fruitful comments from the three independent reviewers which improved the quality and presentation of the paper.

\section{References:}

[1] Bader M.G. and Priest A. M. (1982). Statistical aspects of fiber and bundle strength in hybrid composites, In; hayashi, T., Kawata, K. Umekawa, S. (Eds), Progress in Science in Engineering Composites, ICCM-IV, Tokyo, 1129 - 1136.

[2] Bakouch H. S, Al-Zaharani B., Al-Shomrani A., Marchi V.and Louzad F. (2012). An extended Lindley distribution, Journal of the Korean Statistical Society, 41: 75 -85.

[3] Ghitany M. E., Atieh B. and Nadarajah S. (2008). Lindley distribution and its application, Mathematics and Computers in Simulation, 78: 493-506.

[4] Ghitany M.E., Al-Mutairi D.K., Balakrishnan N. and Al-Enezi L.J. (2013). Power Lindley distribution and Associated Inference, Computational Statistics and Data Analysis, 64: 20 - 33.

[5] Lindley D. V. (1958). Fiducial distributions and Bayes' theorem. Journal of the Royal Statistical Society, Ser. B., 20: 102-107.

[6] Nadarajah S., Bakouch H.S., Tahmasbi R. (2011). A generalized Lindley distribution, Sankhya Series B, 73: 331-359.

[7] Shanker R., Sharma S. and Shanker R., (2013). A two -parameter Lindley distribution for Modeling Waiting and Survival Times Data, Applied Mathematics, 4 (2): 363-368.

[8] Shanker R. and Mishra A. (2013). A two-parameter Lindley distribution, Statistics in Transition New Series, 14(1): 45-56.

[9] Shanker R. and Mishra, A. (2013). A quasi Lindley distribution, African Journal of Mathematics and Computer Science Research, 6 (4) : $64-71$.

[10] Shanker R. and Amanuel A.G. (2013). A new quasi Lindley distribution, International Journal of Statistics and Systems, 8 (2): 143 - 156.

[11] Zakerzadeh H. and Dolati A. (2009). Generalized Lindley distribution, Journal of Mathematical Extension, 3 (2): 13 - 25. 